\title{
Current Techniques and Practices for Myocardial Stress Testing-A Comparative Survey between Malta and International Nuclear Medicine Centres
}

\author{
Karen Borg Grima1, Desiree 0’Leary², Paul Bezzina1', Louise Rainford² \\ ${ }^{1}$ Department of Radiography, Faculty of Health Sciences, University of Malta, Msida, Malta. \\ ${ }^{2}$ Diagnostic Imaging, UCD School of Medicine and Medical Science, University College Dublin, Dublin, Ireland \\ Email: karen.borg-grima@um.edu.mt
}

Received 2 September 2014; revised 2 October 2014; accepted 2 November 2014

Copyright (C) 2014 by authors and Scientific Research Publishing Inc.

This work is licensed under the Creative Commons Attribution International License (CC BY). http://creativecommons.org/licenses/by/4.0/

(c) (i) Open Access

\section{Abstract}

Objectives: The study compared cardiac scintigraphy stress scanning practices applied in a National Maltese Nuclear Medicine centre and in international centres. This was achieved through the design of an online survey which investigated participant knowledge of stress testing, and current procedural practice. Methods: An online survey comprising 12 questions was prepared using Survey Monkey. Professional Nuclear Medicine groups such as the Medical-Physics-Engineering community and Virtual Radiopharmacy were targeted. Access to the survey remained open for eight months during which a periodic reminder was sent to optimise the response rate. Forty-three members responded from across Europe and Australasia. Chi-square tests and comparisons between multiple responses using IBM SPSS 20 were used to evaluate the results. Information related to Maltese practice was collated separately for review and comparative purposes. Results: The online survey participants comprised United Kingdom [72\%], other European countries [18\%] and Australasia [9\%]. The majority of respondents $[n=39]$ reported pharmacological stress testing as being performed either alone or in conjunction with exercise stress testing as the preferred option. Most participants [60\%] were aware of local stress test protocols but had limited knowledge in relation to guidelines designed for cases where patients were not suitable for pharmacological stress testing. Conclusion: The survey provided information about procedures within participating centres for scintigraphic cardiac stress scanning. Differences were identified with regards to the preferred radiopharmaceutical tracers and procedural protocols. Further investigation of examination techniques is warranted, with the aim of increasing standardisation of protocol compliance and the application of more suitable practice. 


\section{Keywords}

\section{Nuclear Medicine Cardiology, Myocardial Stress Test, Coronary Artery Disease, Cardiac Tracers, Pharmacological Stress Agents}

\section{Introduction}

Chronic and noncommunicable diseases, especially cardiovascular disease and cancer, are now leading causes of mortality [1]. Cardiac disease is of increasing concern in Malta; an article in the "Malta Independent", highlighted that $40 \%$ of the total Maltese deaths in 2010 were due to diseases of the circulatory system such as ischaemic heart disease (IHD) [2]. According to the European Cardiovascular Disease statistics published in 2012, CHD was the main cause of mortality amongst Maltese adults in 2010. Mortality rates due to IHD are higher in Malta when compared to other European countries. At least 1069 new angiographic cases are performed yearly in Malta, the majority of whom will be sent for a nuclear medicine cardiac stress scan [3]. The role of nuclear medicine in disease management is becoming more salient. Despite converging needs for nuclear medicine across the developed and developing worlds, there remain key differences between these arenas because of socioeconomic disparities and academic heterogeneity [4]. Reduced access to dedicated NM equipment, such as SPECT cameras [5] may limit cardiac work in these regions as confirmed in a recent survey by the International Atomic Energy Agency [IAEA], which found differences in instrumentation, radiopharmaceuticals and human resource availability [1].

This paper outlines through an online survey that several common trends in practice exist between regions when performing NM myocardial perfusion stress tests. Cardiac tracers, types of cardiac stress tests performed, pharmacological agents used, protocols applied during cardiac stress scanning and the staff injecting the radiopharmaceutical were discussed.

\section{Methods}

Data were collected through an online survey comprising 12 questions in Survey Monkey which was posted on two international websites, the VirRAD and the Medical-Physics-Engineering homepages. The chosen international websites were indicated to the researcher by British and European Nuclear Medicine Societies, as a means for nuclear medicine radiographers of exchanging professional views and information through forums, chat groups or individual discussions for professionals working within the field globally. Questions focussed on: the frequency of myocardial stress examinations, cardiac tracers employed, pharmacological versus exercise stress protocols, pharmacological stress agents, written procedural protocols including staff knowledge and staff responsibilities. Access to the survey was open for 8 months in total for respondents with periodic reminders to encourage greater participation. Additional information related to current practice in Malta was gathered. Data were collated and transcribed to IBM SPSS version 20. Comparisons between multiple responses were carried out using Chi-square tests and cross tabulations. When studying the variances in practice between the regions, the p value was set at a level of significance of 0.05 [5\%].

\section{Results}

Forty-three respondents from centres across Europe and Australasia participated in the online survey. Geographical categorisation following statistician review resulted in 3 regions namely, United Kingdom (UK), rest of Europe and Australasia, for the purpose of comparing the data collected. The majority of participants (72\%) were from the UK, 18\% from various European countries and 9\% from Australasia. The number of cardiac scintigraphy stress scans performed weekly by each of the responding centres is summarised in Table 1. Routine scintigraphic cardiac stress imaging was confirmed as being performed in the national Maltese NM unit, averaging 36 cases per week for the year 2012 [3].

\subsection{Cardiac Tracers}

Literature identifies Technetium-99m (Tc-99m) as the most widely used radionuclide for diagnostic scans being 
performed in developing countries [6]. Similarly the respondents indicated that Technetium-99m labelled cardiac tracers, such as Hexakis [2-methoxy-2-methylpropylisonitrile] (Sestamibi) and [6,9-bis[2-ethoxyethyl]-3, 12-dioxa-6,9-diphosphatetradecane] (Tetrofosmin), are the most commonly used within both European and Australasian regions (Figure 1). The national Maltese NM centre uses Tc-99m Sestamibi. Only 4 centres reported the use of Thallium-201 (Tl-201): Australasia [n = 1], United Kingdom [ $=3]$; with only one of these centres in UK basing its cardiac scintigraphy stress examinations solely on the use of Thallium-201.

\subsection{Pharmacological versus Exercise Stress Testing Protocols}

The online survey identified $64.4 \%$ of responding UK centres as performing scintigraphic cardiac stress imaging using pharmacological vasodilators more frequently than other online survey respondents, as summarised in Figure 2. This is in contrast to the type of stress testing performed weekly for the majority (72\%) of the Maltese examinations, where the use of exercise (treadmill) is preferred.

\subsection{Pharmacological Stress Agents}

The two preferred pharmacological agents of choice identified across the three geographical regions were [ \pm ]-4-[2-[[3-[p-Hydroxyphenyl]-1-methylpropyl]amino]ethyl]-pyrocatechol hydrochloride (Dobutamine) and 6-amino-9- $\beta$-D-ribofuranosyl-9-H-purine (Adenosine) as demonstrated in Figure 3. Maltese practice favoured the use of 2,2',2",2"'-[[4,8-Dipiperidinopyrimido[5,4-d]pyrimidine-2,6-diyl]dinitrilo]-tetraethanol (Dipyridamole). The responses given by the participants with respect to potential contraindications are outlined in Figure 4. Maltese radiographers working in NM indicated the presence of asthma or breathing problems in patients, pa-

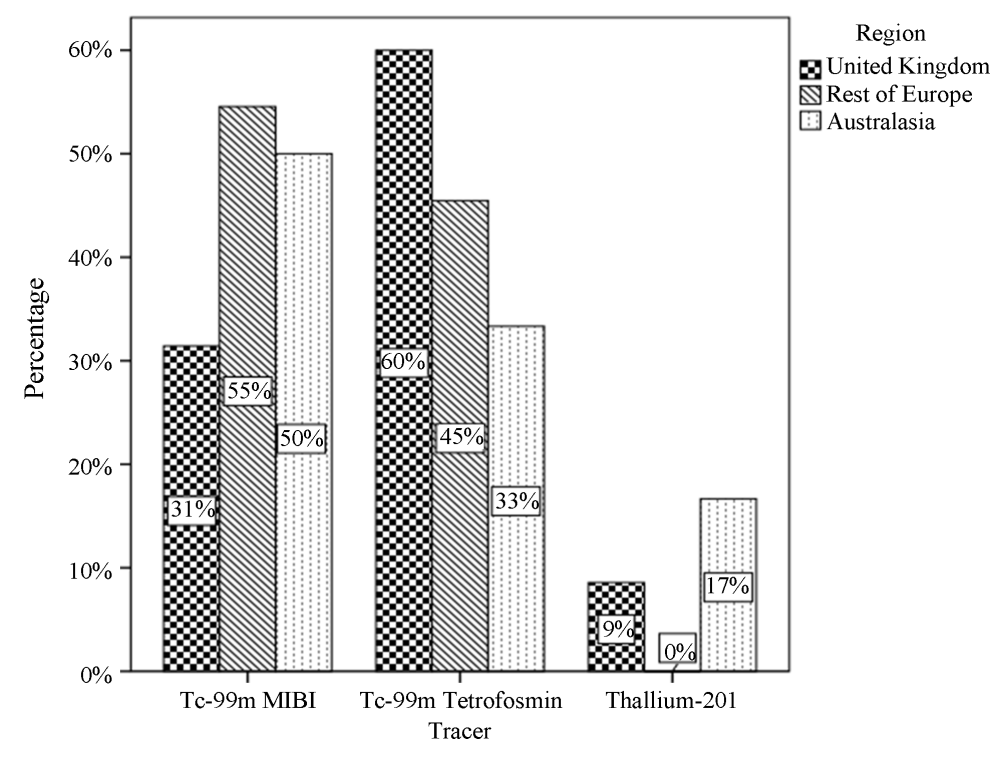

Figure 1. Summary of cardiac tracer usage across the three geographical regions identified by the online survey.

Table 1. The percentage of scintigraphic cardiac stress scans performed weekly emanating from the online survey.

\begin{tabular}{|c|c|c|c|}
\hline \multirow{2}{*}{$\begin{array}{l}\text { Number of } \\
\text { Procedures }\end{array}$} & \multicolumn{3}{|c|}{ Region } \\
\hline & United Kingdom & Rest of Europe & Australasia \\
\hline $1-10$ & $25.8 \%$ & $75.0 \%$ & $25.0 \%$ \\
\hline $11-20$ & $41.9 \%$ & $12.5 \%$ & $25.0 \%$ \\
\hline More than 20 & $32.3 \%$ & $12.5 \%$ & $50.0 \%$ \\
\hline
\end{tabular}

p value 0.106 . 


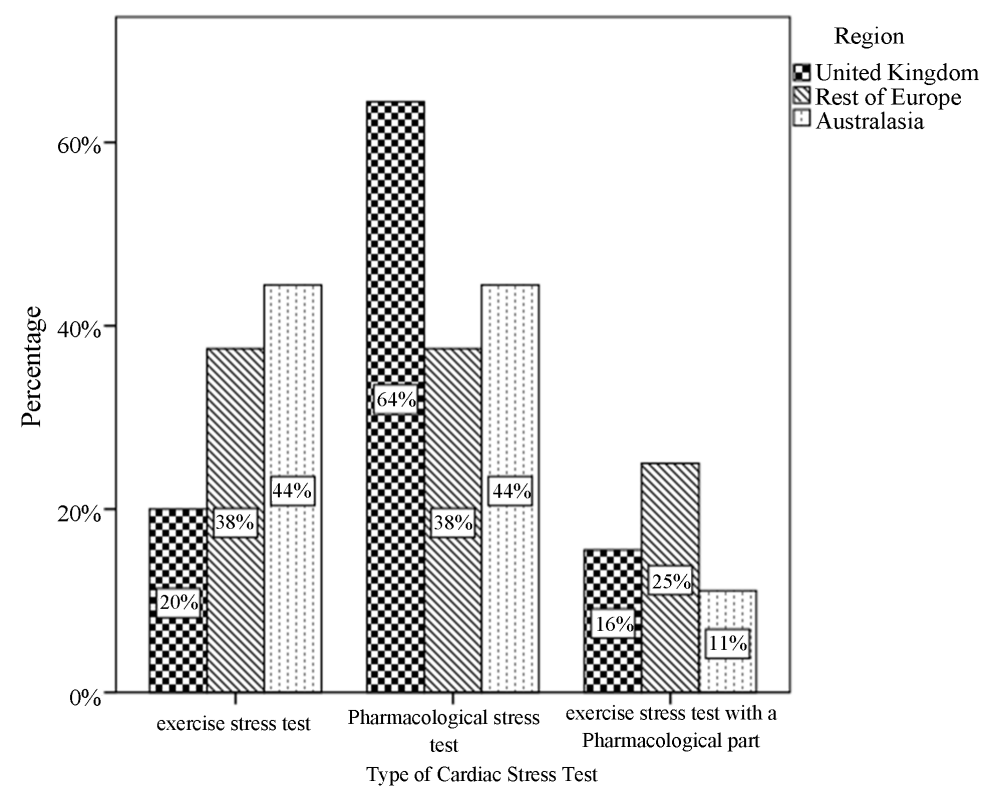

Figure 2. Summary of the types of cardiac stress tests performed per region expressed as a percentage of respondents to the online survey.
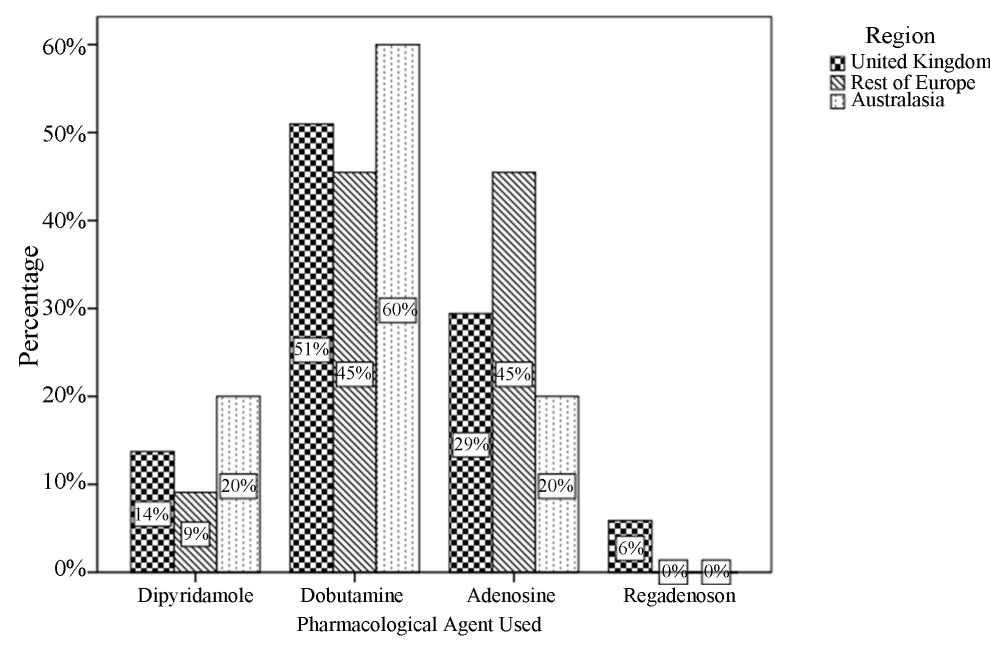
Q United Kingdom P Australasia

Figure 3. Summary of the pharmacological stress agents used per region, expressed as a percentage of respondents to the online survey.

tients being treated with Dipyridamole or taking caffeine products prior to the test, as being the most significant contraindications to the performance of pharmacological stress testing.

\subsection{Protocols Used during Stress Testing}

Participants were asked to confirm the presence of written protocols for scintigraphic cardiac stress scanning, the findings are outlined in Table 2. The staff working in the national Maltese NM unit confirmed the existence of protocols that are used when performing cardiac stress testing.

\subsection{Conducting the Stress Test: Roles and Responsibilities}

In Malta, the ECG stress test component of the myocardial scintigraphy examination is performed by the NM nurse and the ECG technician; an assigned NM Consultant, though not directly present in the room, is always on 
the premises during the cardiac stress testing. Table 3 outlines that in European centres radiopharmaceuticals may be injected either by the nurse or by the radiographer during the stress test; in the UK the radiographer tends to be the lead injector (75.8\% of the participants). Medical doctors inject the radiopharmaceutical in $75 \%$ of the Australasian units. Responses indicate a difference between NM practices across the regions.

All online survey findings were reviewed, descriptive analysis was performed and the responses compared with existing literature and with current practice in Malta. Comparisons between multiple responses were carried out using Chi-square testing. p values higher than the set level of significance were taken to indicate that there was no significant difference between the regions for that particular response.

\section{Discussion}

The IAEA created a NM online database (NUMDAB-http://nucmedicine.iaea.org/details.asp) in 2003, with the aim of investigating current protocol trends across global regions in NM, this site is continually updated. This database represents responses from NM centres in 99 IAEA member states and provides information about the

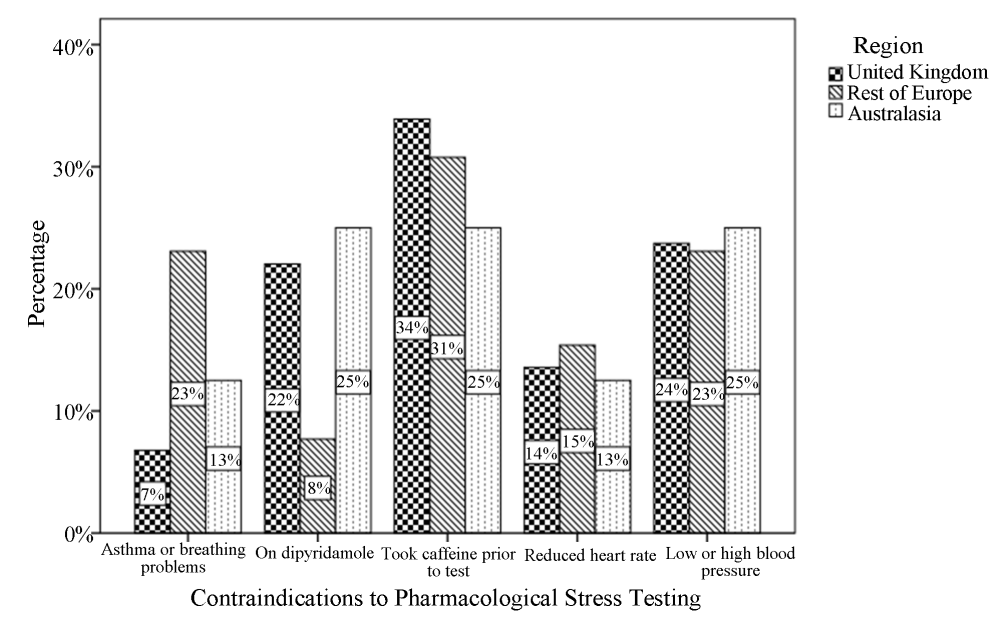

Figure 4. Summary of online survey responses to stated contraindications for pharmacological stress testing, by geographical region.

Table 2. Availability of written procedures, across each region.

\begin{tabular}{ccccc}
\hline & & \multicolumn{2}{c}{ Region } \\
\cline { 2 - 4 } Are written procedures available? & United Kingdom & Rest of Europe & Australasia \\
\hline No & $3.2 \%$ & $0 \%$ & $25.0 \%$ \\
Yes & $64.5 \%$ & $62.5 \%$ & $25.0 \%$ \\
\hline
\end{tabular}

p value 0.255 .

Table 3. Summary of personnel injecting the radiopharmaceuticals for scintigraphic cardiac stress testing, across the geographical regions surveyed.

\begin{tabular}{cccc}
\hline \multirow{2}{*}{ Staff injecting the radiopharmaceutical } & \multicolumn{2}{c}{ Region } \\
\cline { 2 - 4 } & United Kingdom & Rest of Europe & Australasia \\
\hline Doctor or consultant & $6.1 \%$ & $25.0 \%$ & $75.0 \%$ \\
Nurse & $18.2 \%$ & $37.5 \%$ & $0 \%$ \\
Radiographer & $75.8 \%$ & $37.5 \%$ & $25.0 \%$ \\
\hline
\end{tabular}

p value 0.003 . 
extent and type of practice in NM centres worldwide. The NUMDAB currently contains data from 760 Asian NM centres and 50 centres from Western Europe [7]. Only data from one UK NM centre in NUMDAB exist [7].

This online survey in comparison to the NUMDAB received responses from 31 UK centres and these findings therefore are potentially more indicative of practices within the United Kingdom. The response from Asia, in direct contrast to the NUMDAB survey returned a low number of participants [ $\mathrm{n}=2$ ].

NUMDAB figures currently report that 52,287 cardiovascular (CV) scans are performed yearly in the NM centres within Western Europe. This data aligns to the findings of this online survey, which indicated that in 41.9\% of the participating UK centres 11 - 20 cardiac scans were performed weekly (Table 1). In 2012, a total of 1388 Maltese patients underwent stress myocardial perfusion imaging with approximately 36 cases being scanned weekly, which is a higher average figure than for other centres internationally [3].

\subsection{Cardiac Tracers Used}

Despite some disadvantages for usage for both Tc-99m Sestamibi and Tc-99m Tetrofosmin, published literature supports their use for cardiac stress scintigraphy [8] [9]. Sestamibi is taken up mostly by passive cellular diffusion with mitochondrial retention and is then cleared by the hepatobiliary system, explaining liver and intestinal tract activity when this agent is used for cardiac imaging [10]. Tetrofosmin has similar uptake and clearance characteristics to Sestamibi, although it is cleared more rapidly from the liver [10]. A high liver uptake may lead to the false diagnosis of inferior wall ischaemia especially during the rest studies [8]. An alternative tracer is Thallium-201; only four centres reported usage of this option. Literature reports that this cardiac tracer has declined in use due to its less optimal physical characteristics for gamma camera acquisitions when compared to its Technetium-99m counterpart [11]. In some NM centres the use of Thallium-201 is being reconsidered due to the recent supply shortages experienced with Tc-99m [11]. The responses in the online questionnaire indicate that a dual approach was preferred in NM centres currently using Thallium-201, with Thallium being administered for the exercise phase of the clinical examination and technetium based tracers for the resting portion of the examination [12].

Due to the relatively longer physical half-life of Thallium-201 (3.05 days), patients being injected with this tracer have been shown to still have residual biological activity even after 5 half-lives (15.2 days) [13], resulting in an increase to the overall radiation dose received by the patient. Studies have detected the presence of Tl-201 in the urine of patients 25 days post-administration of the tracer and residual Tl-201 activity was noted after 61 days post-administration in one case [14]. This, together with the better imaging characteristics of Technetium99m agents [9] [15], may indicate why Thallium-201 has been replaced in many centres by its Technetium-99m counterparts. Tl-201 may still offer advantages though for myocardial perfusion imaging since research [16] [17] has shown that increased uptake in the lungs after stress imaging with Thallium-201 may be a strong prognostic predictor of CAD.

\subsection{Stress Testing-Pharmacological versus Exercise Protocols}

Literature has shown that $50 \%$ of all myocardial perfusion imaging is performed by pharmacological stress testing rather than exercise methods [18] [19]. Currently, in Malta treadmill electrocardiogram (ECG) stress testing is the norm, with pharmacological stress testing only being adopted in patients who are unable to undergo the former. All Australasian participants stated that pharmacological stress tests would only be performed if the patient's inability to exercise and/or presenting clinical condition prevented exercise stress testing. In contrast to this, the majority of the UK members [71.4\%] replied that pharmacological stress testing would always be carried out on all referred patients. Pharmacological stress testing is preferred over exercise methods in situations where, as reported by the American College of Cardiology/American Heart Association guidelines, contraindications to exercise stress testing exist [20]. The presence of tachyarrhythmias or bradyarrhythmias, and any mental or physical impairment leading to the inability to exercise are all factors that will prevent patients from undergoing exercise stress testing.

Research also suggests that the inability to exercise may be indicative of a poor prognostic outcome [21] [22], and dyspnoea during exercise stress testing has been linked to heart failure and death [23]. Nonetheless concern has been raised identifying the need to investigate further the effects of vasodilators, such as Dobutamine, in patients unable to exercise and having contraindications to pharmacologic stress testing [19]. The triaging of patients prior to their myocardial stress testing is recommended, in order to assure that each patient is undergoing 
the most appropriate stress test, whether pharmacological or exercise induced [24].

\subsection{Pharmacological Stress Agents}

Dobutamine and Adenosine were identified as the preferred pharmacological stress agents in comparison to Dipyridamole and 2-\{4-[[methylamino]carbonyl]-1H-pyrazol-1-yl $\}$ adenosine (Regadenoson) according to the responses from the three participating geographical regions. One explanation for this may be due to the pharmacological characteristics of the drugs. In 51\% of the participating UK centres Dobutamine is the pharmacological stress agent of choice, as it is considered to be safe even in patients with contraindications to pharmacological stress testing [18] [19].

Interesting to note that literature reported a two-fold higher cardiac event rate in patients undergoing Dobutamine stress myocardial perfusion imaging (DSMPI) when compared to the event rates reported after a normal exercise SPECT study [19] [25]; although this may also be attributed to the fact that patients referred for Dobutamine stress testing are a high risk population for cardiac events. Results obtained from canine studies indicated that Dobutamine stress attenuated the myocardial uptake of Tc-99m Sestamibi resulting in an underestimation of myocardial blood flow changes on the obtained myocardial perfusion images, and consequently an overall reduced detection of CAD [25]-[27]. Adenosine, the alternate to Dobutamine which is commonly used, activates nonselectively receptor subtypes: A1, A2B and A3 which may produce short-term side effects in patients. This together with the need to administer Adenosine through a continuous intravenous infusion because of its short biological half-life may discourage the use of such a product for pharmacological stress testing [18].

Regadenoson is a new agent on the market, which unlike Adenosine, targets only A2A receptors, and can therefore be used in patients with mild-to-moderate airway disease and obstructive lung pathology [18]. Based upon the data collected through the online survey, it appears that Regadenoson is being introduced into clinical use within the UK, although its use is still somewhat limited. The ADVANCE trial, the largest study to date, comparing Regadenoson to Adenosine showed very similar incidents of ST-segment changes when using either of these agents, yet less side effects were reported with the former agent [28]. In November of 2013, the FDA issued a report informing health care professionals to screen all nuclear stress test candidates prior to using Regadenoson and Adenosin, to reduce the risks of heart attack associated with the use of these drugs [29].

Through the online survey, the participant's knowledge of contraindications to pharmacological stress testing was also investigated; the answers given by the majority of the respondents suggested uncertainty regarding the use of pharmacological stress agents. Participants were asked which of the options given would be a contraindication to pharmacological stress testing (Figure 4); the answers varied between the three regions but only 3 participants choose the correct option "all of the above".

\subsection{Protocols Used during Stress Testing}

Literature notes that a large variety of cardiac imaging procedures exist across NM and it is the responsibility of individual departments to design suitable protocols [30]. The need for NM radiographers to have a greater understanding of these protocols is also highlighted [30], supported by the deficit in procedure knowledge identified in this study.

\subsection{Conducting the Stress Test: Roles and Responsibilities}

Professional bodies across countries uniformly agree that a physician must be in attendance while performing cardiac exercise stress testing due to the possible related patient complications, even if guidelines pertaining to the performance of this test exist [31]. Literature omits to mention the type of supervision, whether direct or indirect, or the level of risk for which supervision is needed by physicians for such stress tests [31]. In Malta, the ECG and pharmacological stress testing are performed by the NM nurse in conjunction with the ECG technician while the radiographers perform radiopharmacy duties, patient scanning and image processing.

As shown in Table 3, the European participants indicated that the radiopharmaceutical may be injected either by the nurse or by the radiographer during the stress test, depending on who is available at the time.

\section{Limitations}

Due to the uneven geographical spread of results the findings, whilst providing an insight into practices in NM in specific geographical regions, present clustering of participant responses in a limited number of countries. 
This may impact upon the ability to apply the findings to the international population with NM facilities. The information does however provide detail against which current Maltese practice can be compared and offers a good insight into UK practice which was not evident following previous international surveys [7].

Other technical factors play a role in the set-up of a myocardial scintigraphy stress test protocol such as patient position, the type of acquisition set up, the number of ECG leads utilised during the stress test, and also the type of software used to analyse the data acquired. The scope of this survey was to investigate the incidence of common variances and trends present across centres performing myocardial stress testing rather than to assess the technical details in the different protocols that can be used.

\section{Conclusions}

The results of this online survey confirm that several common trends in practice exist between regions when performing NM myocardial perfusion stress tests. Technetium-99m Tetrofosmin appears to be more in use within the UK as is pharmacological stress testing, with Dobutamine being the preferred pharmacological agent in this region. With so many different combinations of tracers, pharmacological agents to choose from and constantly developing new technology, it is essential that guidelines or protocols exist, which leads to more efficient and improved patient care [31]. Although it is performing a relatively high number of cardiac stress examinations on a weekly basis [ $\mathrm{n}=36]$, Malta was noticeably different when compared to the majority of online survey responses in the choice of preferred pharmacological stress agent.

Despite $64.5 \%$ of the UK and $62.5 \%$ of the European survey participants indicating the existence of written procedures for cardiac scintigraphy stress testing, a number of participants in all the 3 regions pointed out that they did not know or were unsure if such written procedures existed. It is critical that procedures and protocols are known, particularly in scenarios where the radiographer is taking on more leading roles in myocardial stress testing. Further research is justified to investigate optimisation of practice for patients attending for cardiac scintigraphic stress test examinations in the national Maltese NM unit.

\section{References}

[1] Dondi, M., Kashyap, R., Paez, D., Pascual, T., Zaknun, J., Bastos, F.M., et al. (2011) Trends in Nuclear Medicine in Developing Countries. Journal of Nuclear Medicine, 52, 16S-23S. http://dx.doi.org/10.2967/jnumed.111.089193

[2] Borg, A. (2013) Almost 60\% Decrease in Heart Disease Deaths in Malta since 1980. The Malta Independent Online.

[3] (2012) Medical Imaging Department Statistics. MID, Mater Dei Hospital, Msida.

[4] Lele, V.R. and Soman, P. (2009) Nuclear Cardiology in India and the Developing World: Opportunities...and Challenges! Journal of Nuclear Cardiology, 16, 348-350. http://dx.doi.org/10.1007/s12350-009-9073-1

[5] Duatti, A. (2008) Promoting Nuclear Medicine in Developing Countries through IAEA Coordinated Research Projects: Technical Reports Series 458 and 459. The Quarterly Journal of Nuclear Medicine and Molecular Imaging, 52, 209211.

[6] Gholamrezanezhad, A., Mirpour, S. and Behbahani, A.A. (2010) Nuclear Medicine in Developing Countries: Perspective from Iran. Journal of Nuclear Medicine, 51, 14N-19N, 22N.

[7] NUMDAB [NUclear Medicine DAtaBase] (2003) International Atomic Agency. http://nucmedicine.iaea.org/details.asp

[8] Goethals, L.R., Santos, I., Caveliers, V., Paulo, A., De Geeter, F., Lurdes, P.G., et al. (2011) Rapid Hepatic Clearance of 99mTc-TMEOP: A New Candidate for Myocardial Perfusion Imaging. Contrast Media \& Molecular Imaging, 6, 178-188.

[9] Kelly, J.D., Forster, A.M., Higley, B., Archer, C.M., Booker, F.S., Canning, L.R., et al. (1993) Technetium-99m-Tetrofosmin as a New Radiopharmaceutical for Myocardial Perfusion Imaging. Journal of Nuclear Medicine, 34, 222-227.

[10] Chamarthy, M. and Travin, M.I. (2010) Altered Biodistribution and Incidental Findings on Myocardial Perfusion Imaging. Seminars in Nuclear Medicine, 40, 257-270. http://dx.doi.org/10.1053/j.semnuclmed.2010.03.005

[11] Gallardo, F.G., Gómez, M.V. and Terol, I. (2011) Prognostic Value of Normal Myocardial Perfusion Scintigraphy with ${ }^{201} \mathrm{Tl}$ in Post-Acute Myocardial Infarction. Revista Española de Medicina Nuclear [English Edition], 30, 83-87. http://dx.doi.org/10.1016/j.remn.2010.09.006

[12] Berman, D.S., Kang, X., Tamarappoo, B., Wolak, A., Hayes, S.W., Nakazato, R., et al. (2009) Stress Thallium-201/Rest Technetium-99m Sequential Dual Isotope High-Speed Myocardial Perfusion Imaging. JACC: Cardiovascular Imaging, 2, 273-282. http://dx.doi.org/10.1016/j.jcmg.2008.12.012 
[13] Geraci, M.J., Brown, N. and Murray, D. (2012) Thallium-201 for Cardiac Stress Tests: Residual Radioactivity Worries Patients and Security. Journal of Emergency Medicine, 43, e439-e442. http://dx.doi.org/10.1016/j.jemermed.2011.05.093

[14] Kol, R., Pelled, O., Canfi, A., Gilad, Y., German, U., Laichter, Y., et al. (2003) The Interference of Medical Radionuclides with Occupational in Vivo Gamma Spectrometry. Health Physics, 84, 756-763. http://dx.doi.org/10.1097/00004032-200306000-00008

[15] Parker, J.A. (2001) Cardiac Nuclear Medicine in Monitoring Patients with Coronary Heart Disease. Seminars in Nuclear Medicine, 31, 223-237. http://dx.doi.org/10.1053/snuc.2001.23529

[16] Gill, J.B., Ruddy, T.D., Newell, J.B., Finkelstein, D.M., Strauss, H.W. and Boucher, C.A. (1987) Prognostic Importance of Thallium Uptake by the Lungs during Exercise in Coronary Artery Disease. New England Journal of Medicine, 317, 1485-1489. http://dx.doi.org/10.1056/NEJM198712103172401

[17] Kaul, S., Finkelstein, D.M., Homma, S., Leavitt, M., Okada, R.D. and Boucher, C.A. (1988) Superiority of Quantitative Exercise Thallium-201 Variables in Determining Long-Term Prognosis in Ambulatory Patients with Chest Pain: A Comparison with Cardiac Catheterization. Journal of the American College of Cardiology, 12, 25-34. http://dx.doi.org/10.1016/0735-1097(88)90351-8

[18] Al Jaroudi, W. and Iskandrian, A.E. (2009) Regadenoson: A New Myocardial Stress Agent. Journal of the American College of Cardiology, 54, 1123-1130. http://dx.doi.org/10.1016/j.jacc.2009.04.089

[19] Navare, S.M., Katten, D., Johnson, L.L., Mather, J.F., Fowler, M.S., Ahlberg, A.W., et al. (2006) Risk Stratification with Electrocardiographic-Gated Dobutamine Stress Technetium-99m Sestamibi Single-Photon Emission Tomographic Imaging: Value of Heart Rate Response and Assessment of Left Ventricular Function. Journal of the American College of Cardiology, 47, 781-788. http://dx.doi.org/10.1016/j.jacc.2005.09.059

[20] Gibbons, R.J., Balady, G.J., Timothy Bricker, J., Chaitman, B.R., Fletcher, G.F., Froelicher, V.F., et al. (2002) ACC/AHA 2002 Guideline Update for Exercise Testing: Summary Article: A Report of the American College of Cardiology/American Heart Association Task Force on Practice Guidelines (Committee to Update the 1997 Exercise Testing Guidelines). Journal of the American College of Cardiology, 40, 1531-1540. http://dx.doi.org/10.1016/S0735-1097(02)02164-2

[21] Myers, J., Prakash, M., Froelicher, V., Do, D., Partington, S. and Atwood, J.E. (2002) Exercise Capacity and Mortality among Men Referred for Exercise Testing. New England Journal of Medicine, 346, 793-801. http://dx.doi.org/10.1056/NEJMoa011858

[22] Roger, V.L., Jacobsen, S.J., Pellikka, P.A., Miller, T.D., Bailey, K.R. and Gersh, B.J. (1998) Prognostic Value of Treadmill Exercise Testing: A Population-Based Study in Olmsted County, Minnesota. Circulation, 98, $2836-2841$. http://dx.doi.org/10.1161/01.CIR.98.25.2836

[23] Bernheim, A.M., Kittipovanonth, M., Scott, C.G., McCully, R.B., Tsang, T.S. and Pellikka, P.A. (2009) Relation of Dyspnea in Patients Unable to Perform Exercise Stress Testing to Outcome and Myocardial Ischemia. American Journal of Cardiology, 104, 265-269. http://dx.doi.org/10.1016/j.amjcard.2009.03.028

[24] Rao, S., Lele, V. and Lele, R.D. (2005) Prognostic Value of 99mTc-Sestamibi Stress Myocardial Perfusion Single Photon Emission Computed Tomography (SPECT) in Ischemic Heart Disease. Journal of the Association of Physicians of India, 53, 1036-1042.

[25] Calnon, D.A., McGrath, P.D., Doss, A.L., Harrell Jr., F.E., Watson, D.D. and Beller, G.A. (2001) Prognostic Value of Dobutamine Stress Technetium-99m-Sestamibi Single-Photon Emission Computed Tomography Myocardial Perfusion Imaging: Stratification of a High-Risk Population. Journal of the American College of Cardiology, 38, 1511-1517. http://dx.doi.org/10.1016/S0735-1097(01)01565-0

[26] Calnon, D.A., McGrath, P.D., Doss, A.L., Watson, D.D. and Beller, G.A. (1998) Prognostic Value of a Normal Dobutamine Stress Tc-99m Sistamibi SPECT Study. Journal of the American College of Cardiology, 31, 167. http://dx.doi.org/10.1016/S0735-1097(98)81372-7

[27] Zahid, M., Kapila, A., Eagan, C.E., Yusko, D.A., Miller, E.D. and Missenda, C.D. (2013) Prevalence and Significance of Electrocardiographic Changes and Side Effect Profile of Regadenoson Compared with Adenosine during Myocardial Perfusion Imaging. Journal of Cardiovascular Disease Research, 4, 7-10. http://dx.doi.org/10.1016/j.jcdr.2012.10.001

[28] FDA (2013) Lexiscan [Regadenoson] and Adenoscan [Adenosine]: Drug Safety Communication—Rare but Serious Risk of Heart Attack and Death. Drug Safety Communication, White Oak.

[29] Mann, A. and White, M.P. (1998) Role of the Technologist in Nuclear Cardiology. Journal of Nuclear Cardiology, 5, 438-441. http://dx.doi.org/10.1016/S1071-3581(98)90150-7

[30] Zecchin, R.P., Chai, Y.Y., Roach, K.A., Speerin, R., Lindsay, G., Squire, J. and Denniss, A.R. (1999) Is Nurse-Supervised Exercise Stress Testing Safe Practice? Heart \& Lung: The Journal of Acute and Critical Care, 28, 175-185. 
http://dx.doi.org/10.1016/S0147-9563(99)70057-9

[31] Helfant, R.H., Klein, L.W. and Agarwal, J.B. (1987) Role of Cardiac Testing in an Era of Proliferating Technology and Cost Containment. Journal of the American College of Cardiology, 9, 1194-1198.

http://dx.doi.org/10.1016/S0735-1097(87)80327-3 
Scientific Research Publishing (SCIRP) is one of the largest Open Access journal publishers. It is currently publishing more than 200 open access, online, peer-reviewed journals covering a wide range of academic disciplines. SCIRP serves the worldwide academic communities and contributes to the progress and application of science with its publication.

Other selected journals from SCIRP are listed as below. Submit your manuscript to us via either submit@scirp.org or Online Submission Portal.
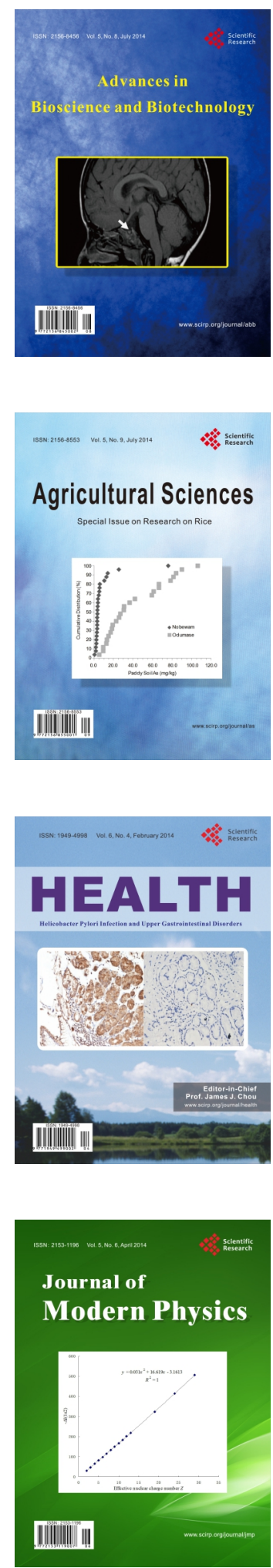
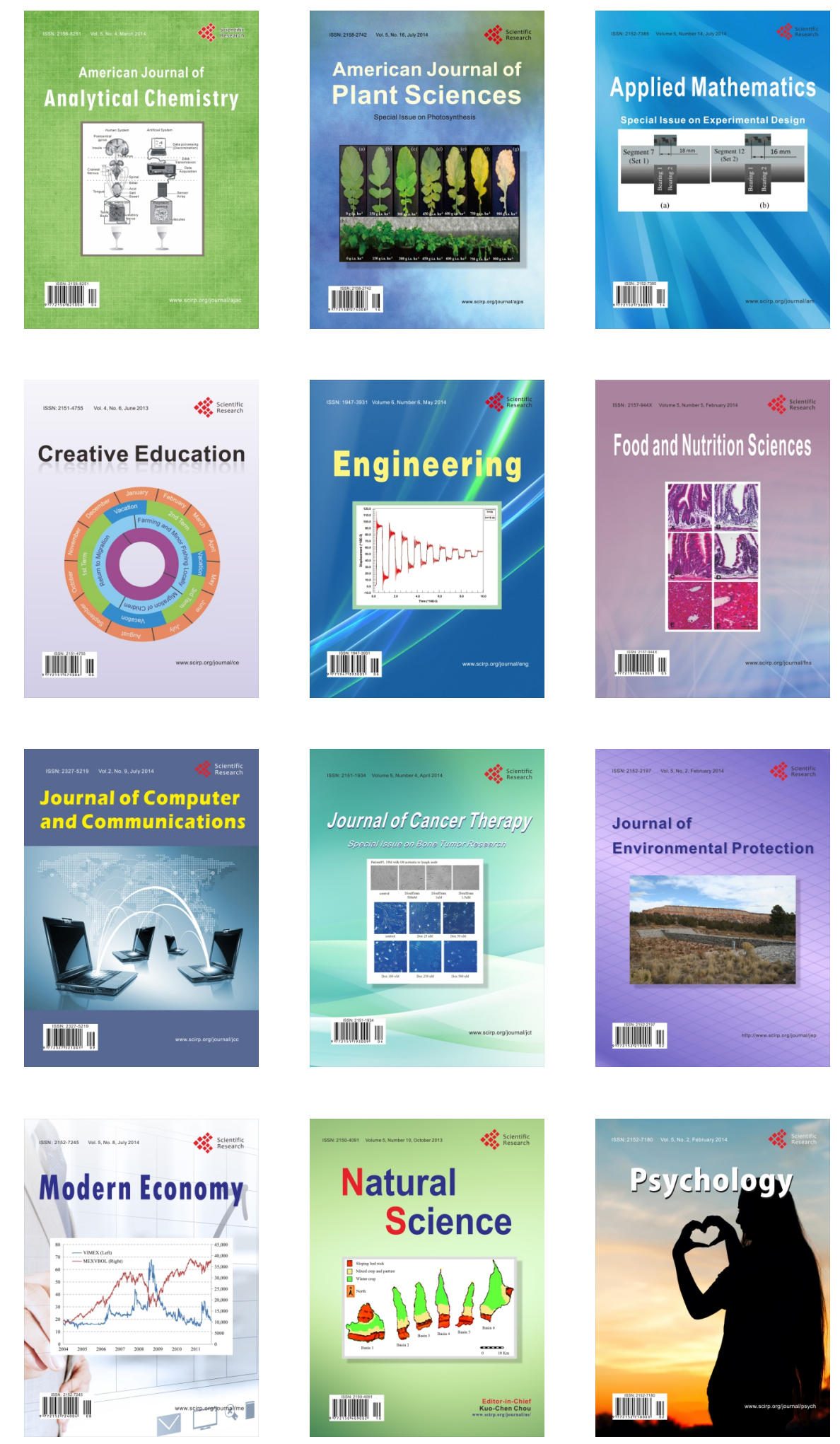